\section{Integrative Medicine \\ International}

\title{
Targeting Neurogenesis: A Promising Therapeutic Strategy for Post-Stroke Treatment with Chinese Herbal Medicine
}

\author{
Jiangang Shen ${ }^{a, b}$ Xingmiao Chen ${ }^{a, b} \quad$ Xi Chen $^{a}$ Ruixia Deng ${ }^{a, b}$ \\ aSchool of Chinese Medicine, LKS Faculty of Medicine, and besearch Centre of Heart, Brain, \\ Hormone and Healthy Aging, The University of Hong Kong, Hong Kong, SAR, China
}

Key Words

Neural stem cells $\cdot$ Chinese herbal medicine $\cdot$ Neurogenesis $\cdot$ Stroke

\begin{abstract}
Recent progress suggests that neural stem/progenitor cells can potentially develop into new functional neurons in adult brain, offering hope for regeneration therapies for stroke treatment. Targeting adult neurogenesis becomes a novel and promising therapeutic strategy for brain repair and recovery of neurological functions. Traditional Chinese Medicine (TCM) has a long history with accumulated experiences and case reports using herbal formulas to treat stroke disability. The combination of Chinese herbal medicine and stem cell biology approaches provides great potential for post-stroke rehabilitations. In the last decade, large efforts have been made to investigate the molecular targets for the regulation of adult neurogenesis and to explore the active compounds and molecular targets of herbal medicine for regeneration therapy. Herein, we reviewed the current progress concerning the molecular targets and cellular signaling pathways involved in adult neurogenesis after cerebral ischemia. We then briefly introduced Chinese medical theory and herbs for stroke treatment in TCM. Finally, we reviewed the current knowledge about the effects of Chinese herbal formulas, active fractions and active compounds on promoting adult neurogenesis as well as their molecular targets. Although the precise mechanisms and molecular targets of herbal medicine for neurogenesis are still unclear, current progress at least provides a cue for exploring the therapeutic principles of Chinese herbal medicine and developing new drugs for brain repair after stroke.
\end{abstract}




\section{Background}

Stroke is the second most common cause of death and a leading cause of adult disability in human diseases worldwide [1, 2]. In China, the age-adjusted stroke prevalence ranges from 259.86 to 719 per 100,000 people per year [3]. With increasing life expectancy, it is anticipated that stroke will become a major burden in public health worldwide.

Stroke involves a heterogeneous group of processes. Ischemic stroke accounts for about $85 \%$ of all stroke cases, while hemorrhagic stroke accounts for the remaining $15 \%$ of all stroke cases [4]. Ischemia is defined as a reduction in blood flow sufficient to result in an almost immediate lack of oxygen and glucose in the brain tissue. The brain is exquisitely sensitive to ischemia, such that even brief ischemic periods to neurons can initiate a complex sequence of events that may ultimately result in cell death [5]. Following neuronal cell death due to cerebral ischemia, the rapid degeneration of brain structure could induce severe neurological dysfunctions. The clinical symptoms of ischemic stroke include paralysis or numbness of one side of the body, loss of speech and vision, and trouble with balance or coordination. Early restoration of blood flow is extremely important in order to limit brain injury after stroke.

In the last decades, tremendous efforts have been made to develop therapeutic approaches for stroke treatment. Nevertheless, the clinical outcome is still not satisfying. Recombinant tissue plasminogen activator is an FDA-approved drug, but it has a critical time window within $4.5 \mathrm{~h}$ with the potential risk of hemorrhagic transformation. Although thrombolytic therapy has decreased the morbidity and mortality of stroke [6], most stroke patients could not catch up on the golden therapeutic window beginning from their initial clinical symptoms in order to reach a definite diagnosis. While many neuroprotective drugs are effective in animal models, they failed to pass clinical trials [7]. Thus, the development of novel therapeutic strategies and new drugs is important for stroke treatment. Therefore, post-stroke rehabilitation becomes a major therapeutic focus for most post-stroke patients. Unfortunately, the currently available therapies are only rarely successful in improving recovery from neurological deficits.

The discovery of adult neurogenesis sheds light on the development of new therapeutic approaches for stroke. Neural stem/progenitor cells (NSCs) can potentially develop into new functional neurons in the adult central nervous system (CNS) $[8,9]$. Adult neurogenesis mainly occurs in the subgranular zone of the dentate gyrus of the hippocampus and the subventricular zone (SVZ) adjacent to the lateral ventricle [10,11]. Enhanced neurogenesis was found in hypoxic NSCs in vitro [12,13] and in ischemic brains of neonatal mice [14], adult rats [15] and aged humans in vivo [16]. Enhanced neurogenesis either by stem cell transplantation or stimulation of endogenous neurogenesis could partly amend the damaged brain functions, raising hopes for brain repair treatment. Recent progress in stem cell therapy proposes the approach of transplantation of NSCs targeting brain repair [17, 18]. Experimentally, the transplantation of NSCs is a promising strategy for the replacement of dead or injured neurons. Nevertheless, many remaining problems, such as ethical controversial, standardization, viability, purity of cell materials and safety issues, greatly block the clinical application at least in the near future. Thus, most efforts focus on stimulating the formation of and preventing the death of neurons and glial cells in the CNS.

\section{Current Molecular Targets for Adult Neurogenesis}

NSCs have limited capacities for growth, differentiation and generation of new neurons in order to repair the damaged CNS in adults [8,9]. At 1-2 weeks after transient global ischemia, newly formed cells migrate into the granule cells and promote functional recovery 
[19]. However, this spontaneous brain repair seems insufficient to amend neurological deficits in most stroke cases. The major obstacles include: (a) most of the newly proliferated NSCs are unable to form new functional neurons and integrate into the neurological network and (b) there is poor survival of new neuroblasts after 4 weeks of stroke [19]. To overcome those problems, pharmacological manipulation targeting NSCs is proposed for stimulating neurogenesis and promoting the recovery of neurological deficits. For example, treatment with basic fibroblast growth factor (bFGF) and epidermal growth factor (EGF) can stimulate massive regeneration and trigger brain repair after stroke [20].

NSCs within the adult brain germinal centers reside in a specialized microenvironmental niche. They are in close proximity to blood vessels and are surrounded by glial cells to form a microenvironmental niche. The proliferation and differentiation of NSCs and neural growth depend on the microenvironmental niche signals, which include bFGF, EGF, vascular endothelial growth factor (VEGF) and nerve growth factor among others [21-24]. Among them, VEGF plays a critical role in neural regeneration. It exerts its action via phosphotyrosine kinase receptors VEGFR1 and fms-like tyrosine kinase. VEGF can stimulate the proliferation and differentiation of NSCs and neurogenesis in vitro and in vivo [22, 24]. It was reported that VEGF enhanced neurogenesis, neuromigration and angiogenesis, and improved neurological functions in the ischemic brain and spinal cord injury [24-27]. VEGF functions as a niche signal for the proliferation of NSCs in response to the bFGF signal and mediates cross-talk between NSCs and endothelial cells in the niche [28-30].

Notch signaling plays a critical role in the maintenance, proliferation and differentiation of NSCs in the microenvironmental niche [31]. Notch proteins mediate multiple cellular functions through cell-cell interactions. Upon ligand binding, Notch is cleaved and releases a notch intracellular domain (NICD). NICD represses or activates transcription factors. Notch signaling can promote NSC proliferation and modulate glial and neural fates in a stepwise manner, first by inhibiting neuronal fate and promoting glial fate and second by inducing astrocyte differentiation. The Notch-mediated inhibition of neuronal differentiation is achieved by preventing the neighboring cells from becoming the same cell type. Focal cerebral ischemia activates Notch1 signaling and promotes the proliferation of NSCs in the SVZ of adult brains [31, 32]. The upregulation of Notch1 signaling, including Notch1, NICD and hairy and enhancer of split (Hes)1, can promote the proliferation of the NSCs and inhibit neuronal differentiation in ischemic brains [33, 34]. The Notch1 signal mediates the crosstalk of NSCs and endothelial cells in the microenvironmental niche. Endothelial cells can release soluble factors to stimulate the self-renewal of NSCs and inhibit their differentiation via activating Notch and Hes1 $[35,36]$. The cross-talking of VEGF and Notch signaling can promote the tissue regeneration [36].

The Wnt/ $\beta$-catenin pathway is another important regulator for neurogenesis and oligodendrogenesis. Wnt proteins are involved in multiple processes during the CNS development, including cell proliferation, migration, specification and differentiation. $\beta$-Catenin is a key downstream effector of Wnt. Activation of $\beta$-catenin could promote the proliferation of the neural progenitor pool. The Wnt/ $\beta$-catenin pathway regulates the differentiation of neural progenitor cells into neurons and delays oligodendrocyte development [37,38]. Wnt signaling contributes to the functional recovery after ischemic injury by increasing neurogenesis or neuronal survival in the striatum after focal ischemic injury [39].

The sonic hedgehog (Shh) signaling pathway also regulates neurogenesis [40]. Shh acts through a receptor complex composed of PTCH and SMO for the activation of the target gene Gli [41]. Shh promotes hippocampal progenitor cell proliferation [42]. Disruption of Shh signaling by conditional knockout of its downstream mediator SMO resulted in the reduction of NSC proliferation [43]. Shh can also regulate migration. Inhibiting Shh in the adult SVZ prevented neuroblasts (type A cells) from migrating to the olfactory bulb [44]. 
Our recent works explored the roles of caveolins (Cav) in the regulation of neurogenesis. Caveolins are a group of 22-kDa structural proteins including Cav-1, Cav-2 and Cav-3. Cav-1 and Cav-2 are widely expressed in neuronal cell types and brain regions [45-47], whereas Cav-3 is muscle specific [48]. Caveolins interact with proteins via the caveolin scaffold domain [49]. They negatively regulate a variety of signal pathways, such as G proteins, nitric oxide synthases, Src tyrosine kinases, ras, estrogen receptors, protein kinase $C$, integrins, MAP kinase and EGF-R [50-52]. Cav-1 decreased neurite outgrowth and branching, and neurite density in injured differentiated PC12 cells [53] and blocked the formation of neurites and phosphorylation of extracellular signal-regulated kinase (ERK) upon bFGF treatment in N2a cells [54]. We found that Cav-1 knockout mouse brains displayed an increased proliferation and differentiation of adult NSCs in the SVZ area [55-57]. The major discoveries include: (1) Cav-1 promoted astroglial differentiation of NSCs through modulating Notch1/NICD and Hes1 expressions [55]; (2) Cav-1 inhibited oligodendroglial differentiation of NSCs through modulating $\beta$-catenin expression [56], and (3) Cav-1 inhibited neuronal differentiation via downregulations of VEGF, p44/42 MAPK, Akt and Stat3 signaling pathways. Downregulation of Cav-1 contributed to hypoxia-mediated neuronal differentiation in neural progenitor cells [57]. Therefore, Cav-1 plays a critical role in neural progenitor cell proliferation and cell fate decision in the post-stroke brain.

Phosphatidylinositol 3-kinase (PI3K)/Akt and ERK signaling pathways are also involved in the process of neurogenesis. They can regulate hypoxia-inducible factor (HIF)- $1 \alpha$, GSK-3 $\beta$, and cAMP response element-binding protein (CREB). HIF-1 $\alpha$ deletion was reported to impair hippocampal Wnt-dependent processes, including NSC proliferation, differentiation and neuronal maturation [58]. GSK-3 $\beta$ can function as signaling nodes to regulate and orchestrate the diverse cellular responses in neurogenesis via affecting HIF- $1 \alpha, \mathrm{HIF}-2 \alpha$ and $\beta$-catenin. CREB is required for EGF-induced cell proliferation and serum response element activation in NSCs of adult mouse brain [59, 60]. Vanadium, a stimulator of PI3K/Akt and ERK, increased NSC proliferation and promoted the migration of newborn neurons in the ischemic brain [61]. PI3K/Akt and ERK also regulate many mitogenes including bFGF, Shh, and insulin-like growth factor 1 . Thus, PI3K/Akt and ERK signaling pathways are important targets for drug discovery for adult neurogenesis.

A recent review article summarized the major cellular signaling pathways in adult neurogenesis: Shh, miR-124, Sox2, Tlx and Wnt/ $\beta$-catenin signaling pathways are regulators for cell proliferation; basic helix-loop-helix transcription factors such as Asc1, Neurog2 and Tbr2 and epigenetic factors like Gadd45b, MBD1, MeCP2 and Mll1 are necessary for neuronal differentiation and maturation; insulin-like growth factor 1 and Shh are essential for neuroblast migration; extrinsic factors including BDNF, FGF-2, GABA, glutamate and NT-3 play important roles in regulating neuronal survival and dendritic arborization, synaptic plasticity and synapse formation; intrinsic factors including DISC1, Klf-9, NeuroD1, Cdk and CREB participate in neuronal survival, dendritic arborization, synaptic integration and maturation [62].

In summary, adult neurogenesis is a multistep process that requires the proliferation of NSCs, the differentiation into the specific neuronal cell types, migration, and that the new cells differentiate, survive and integrate into existing neural networks. Signaling pathways involved in the regulations of adult neurogenesis are very complex. The signal molecules not only come from the NSCs themselves but also from the microenvironmental or neurogenic niche. Therefore, targeting critical cellular signaling pathways is an important therapeutic strategy for drug discovery for brain repair for post-stroke treatment. 


\section{Classic Concepts and Representative Therapeutics for Stroke in Traditional Chinese Medicine}

With the progress of stem cell biology, we can expect that more and more cellular signaling pathways will be discovered, making the underlying mechanisms of brain repair complicated. The question arises as how to find a 'magic drug' which can regulate overall profiles of cellular signaling and genes for neurogenesis. Given that Chinese herbal medicines generally include multiple herbal items and therapeutically target multiple signaling pathways, Chinese herbal medicine therapy may provide a solution to regulate the complex network systems to promote brain repair. Since Chinese herbal medicine has been used for thousands of years and produced a huge amount of case reports, intensive investigations on the neurogenesis-promoting effects of the active compounds, individually and synergistically, and their related molecular targets would create new directions for drug discovery to promote neurogenesis in poststroke studies.

In Traditional Chinese Medicine (TCM) theory, stroke is named as 'wind stroke' with the definition of a condition mainly characterized by sudden collapse and loss of consciousness, deviation of the tongue and mouth, hemiplegia, slurred speech, or only deviation of the tongue and mouth and hemiplegia without collapses. According to TCM concepts, 'wind stroke' is caused by a long-term exposure to multiple pathological conditions including abnormal diet and lifestyles, emotional stress, abnormal psychological stress, and constitution factors, resulting in the deficiency of $q i$ and abnormal movement of $q i$ and blood. The patients with a particular constitution named deficiency of gan-ying and shen-ying is susceptible to pathological factors and easy to be attacked by 'wind stroke'. The attack of 'wind stroke' could be attributed to the reversed flow of $q i$ and blood, and subsequently produce wind, fire, phlegm and blood stasis, inducing the formation of cerebral thrombosis or cerebral hemorrhage. According to the patient's clinical conditions, 'wind stroke' can be divided into three subtypes: 'meridian stroke', 'zhang-fu stroke' and sequela. 'Meridian stroke' is considered as the mild one, but 'zhang-fu stroke' is the most severe clinical pattern, characteristically with obnubilation. Accordingly, different therapeutic approaches and formulas are specifically designed to treat different TCM clinical patterns based on TCM syndrome differentiation. Based on the histological descriptions and clinical observations, the clinical patterns of 'meridian stroke' in TCM are generally equivalent to the clinical characteristics of primary hypertension and transient ischemic attack. Tianma gouteng decoction and zhengang xifen decoction are representative formulas for 'meridian stroke' treatment. As for 'zhang-fu stroke', its clinical pattern is similar to acute ischemic stroke or hemorrhagic stroke with serious brain damage showing clinical symptoms of obnubilation or coma. 'Zhang-fu stroke' has two clinical subtypes including excessive syndrome and deficiency syndrome. Angong niuhuang wan and lingjiao guoteng decoction are two representative formulas for 'excessive syndrome with gang-yang and gang wind hyperactivity and upwards', whereas shenfu decoction is particularly designed for the 'zhang-fu stroke' with the deficiency of yang qi. For sequela, jieyu dan is a representative formula for relieving the symptoms of deviation of the tongue and mouth, hemiplegia and slurred speech. This clinical pattern is generally considered as the syndrome of phlegm and blood stasis. Another representative formula is buyang huanwu decoction (BYHWD). This formula is specifically designed for neurological deficits and dysfunctions with the types of $q i$ deficiency-induced blood stasis.

In recent years, large efforts have been made to understand the therapeutic principles, molecular targets and active compounds of the TCM herbal formulas. For example, by using experimental stroke animal models and conducting clinical trials, tianma gouteng decoction, a representative formula for the treatment of primary hypertension and transient ischemic attack, revealed to decrease blood pressure, ameliorate cognitive impairment and protect 
neural cells in ischemic brains [63-65]. Due to the poor design of clinical trials, however, a recent systematic review challenged its clinical efficacy for the treatment of primary hypertension [66]. BYHWD is one of the classic formulas for post-stroke disability with intensive investigations. Several recent systematic reviews and meta-analysis studies conducted by us and others provide experimental and clinical evidence to support the application of BYHWD in the post-stroke treatments $[67,68]$. Although the quality of clinical trials needs to be improved, the effects of this formula on improving neurological functions have been well accepted. The active constitutions and the metabolites of BYHWD have been reported as well [69, 70]. In the following sections, we will focus on the current progress in the studies of Chinese herbal medicine for promoting neurogenesis and improving neurological functions by using different in vivo and in vitro experimental systems, providing opportunities for post-stroke treatment.

\section{Current Progress in Chinese Herbal Medicine for Promoting Neurogenesis and Their Related Molecular Targets}

In the last decades, great efforts have been made to investigate the effects of Chinese herbal medicine on promoting neurogenesis and their related molecular targets. In TCM practice, therapeutic approaches are generally designed based on unique TCM theory and the prescriptions include multiple herbs; the items can be changed with the different clinical syndrome patterns during the different phases of a disease. From the angle of photochemistry, the TCM formulas generally consist of thousands of chemical ingredients which may target multiple cellular signaling pathways. With comprehensive and advanced analytic approaches, scientists are trying to explore the therapeutic principles of TCM formulas by identifying their active compounds and molecular targets involved in the regulation of neurogenesis. Herein, we summarize and review the current progress in those aspects.

\section{Representative TCM Formulas and Their Molecular Targets for Neurogenesis}

\section{Buyang Huanwu Decoction}

BYHWD has been a classic TCM formula for post-stroke disability treatment for 300 years. The formula was first introduced in a renowned TCM textbook named Yilin Gaicuo (Correction on Errors in Medical Books) by Dr. Wang Qing-ren in the Qing Dynasty. According to Dr. Wang's opinion, paralysis caused by ischemic stroke is similar to the loss of 'five of ten' due to qi deficiency and blood stasis. Thus, boosting the $q i$ and restoring the blood circulation are the key points to treat this clinical pattern of ischemic stroke. BYHWD consists of 7 items including Astragalus membranaceus, Angelica sinensis, Paeonia lactiflora, Ligusticum chuanxiong, Carthamus tinctorius, Prunus persica and Lumbricus at a ratio of 120:4.5:3:3:3:3:3. A. membranaceus is the dominating herb in this formula, which accounts for about $85 \%$ of the whole decoction. It functions as the 'king' component in the formula to invigorate $q i$ and enhance yang-qi. A. sinensis, P. lactiflora, L. chuanxiong, C. tinctorius and P. persica are 'minister' and 'assistant' components used to promote blood circulation and replenish blood. Lumbricus is used as a 'guiding' component to smooth the movement of $q i$ and blood in the channels. Combining these 7 items together, BYHWD can enhance the effect of boosting qi and removing the blood stasis. Several clinical trials have been conducted to evaluate the efficacy of BYHWD for post-stroke disability. BYHWD revealed to promote neurogenesis, reduce infarction volume and improve neurological functions in post-stroke animal models and human subjects [71-75]. Herein, we summarized the current research progress in the pharmacological activities of BYHWD and its ingredients and reviewed its potentials for post-stroke rehabilitation. 
Neuroprotective Effects and Its Molecular Targets

Reactive oxygen species are important pathological factors in cerebral ischemia/reperfusion injury. Our previous studies showed that BYHWD inhibited the neuronal nitric oxide synthase activity and protected neurons from oxidative injury in a permanent focal cerebral ischemia model [76]. Both in vitro and in vivo studies revealed that BYHWD had anti-apoptotic effects against cerebral hypoxic/ischemia reperfusion injury. The in vitro studies showed that BYHWD protected cortical neurons from hypoxia-induced apoptotic cell death through scavenging $\mathrm{NO}$ and superoxide $\left(\mathrm{O}_{2}^{-}\right)$, downregulating the expressions of $\mathrm{p} 53$ and p21 genes and upregulating the expression of bcl-2 $[77,78]$. The in vivo studies showed that oral administration of BYHWD reduced the number of TUNEL-positive neurons by attenuating the expression of caspase-3 p20, a product of catalytically active caspase-3 in the hippocampal CA1 region [79]. BYHWD obviously inhibited the expression of HSP70 mRNA but had no effect on the HSP70 protein [80]. BYHWD revealed to modulate pro-inflammatory mediators including IL-1 $\beta$ and TNF- $\alpha$ in an experimental cerebral ischemia stroke model [81]. The active fractions and compounds, including alkaloid, glycoside, polysaccharide and aglycone, were found to inhibit inflammatory cytokines, alleviate the inflammatory reactions and downregulate the expression of caspase- 1 in cerebral ischemia/reperfusion injury. The alkaloid, glycoside and aglycone fractions inhibited the expression of caspase-3 in the hippocampus, cortex and medulla and protected neurons from cerebral ischemia injury [82]. $\mathrm{Ca}^{2+}$ overload and excitatory amino acid are important mediators of neurotoxicity in cerebral ischemia/ reperfusion injury. BYHWD showed to reduce intracellular $\mathrm{Ca}^{2+}$ concentration in neural progenitor cells [83] and regulate the metabolism of endothelin and calcitonin gene-related peptide in patients with early cerebral infarction [74]. A recent study reported that BYHWD treatment significantly decreased the level of excitatory amino acids and increased inhibitory amino acids in cerebrospinal fluid extracted from the rats subjected to cerebral ischemia/ reperfusion injury [84].

In addition, the effects of BYHWD on the antithrombotic functions were also studied by using human umbilical vein perfusion. BYHWD reduced the von Willebrand factor release and inhibited the conversion of fibrinogen to fibrin catalyzed by thrombin [85]. Administration of BYHWD increased the blood flow in the hippocampal region after occlusion and inhibited the hypoperfusion after reperfusion in ischemia/reperfusion rats [86]. In addition, BYHWD reduced the platelet activator factor in the arterial blood after thrombosis [87]. Therefore, the neuroprotective mechanisms of BYHWD are related to regulate multiple cellular signaling pathways.

\section{Neurogenesis-Promoting Effects and Molecular Targets}

Our early studies revealed that BYHWD containing serum significantly promoted the proliferation of neurons under both normal and hypoxic conditions [88]. Further studies found that BYHWD increased the 5-bromo-2-deoxyuridine-positive neural progenitor cells in the rat hippocampus and SVZ after ischemic stroke, suggesting that BYHWD could improve the neural progenitor proliferation [72]. Furthermore, BYHWD treatment also stimulated the differentiation of neural progenitor cells as evidenced by increasing the neurofilamentpositive cells and glial fibrillary acidic protein-positive cells in cultured neural progenitor cells [83]. A recent study reported that BYHWD treatment significantly increased 5-bromo2-deoxyuridine-positive cells in the SVZ, subgranular zone and corpus striatum of the infarcted brain by upregulating the expression of migration activators including stromal cell-derived factor 1, CXC chemokine receptor 4, VEGF, Reelin and BDNF in the ipsilateral infarct area after middle cerebral artery occlusion cerebral ischemia. In addition, BYHWD treatment was able to promote the neuronal differentiation, which closely related to the migratory process of neural progenitor cells in middle cerebral artery occlusion rats. These results suggest that 
BYHWD might promote the migration of neural progenitor cells to the ischemic brain area [89].

VEGF and its receptor Flk1 are important neurotrophic, neuroprotective and neuroproliferative factors. Our study showed that BYHWD upregulated the expressions of VEGF and Flk1 at the SVZ and cortex in the post-ischemic brains [72]. Further studies revealed that BYHWD treatment increased the expression of VEGF in the serum of stroke patients [90]. Moreover, the combination of mesenchymal stem cell transplantation and BYHWD treatment repaired the injured blood vessels and lesion tissues by inducing the expression of VEGF and Ki-67 [91].

Moreover, a genomic assay was applied to explore the regulation of BYHWD on overall genomic profiles in the post-stroke brains. Treatment of BYHWD remarkably led to upregulate 25 genes but downregulate 6 genes in the brain tissues of a rat model of experimental cerebral ischemia [92]. A recent study revealed that BYHWD treatment upregulated the expressions of 93 genes but downregulated 284 genes in a cerebral ischemia mouse model. Among the 93 genes, 6 are associated with neurogenesis and 9 are related to nervous system development. In addition, BYHWD also showed to regulate the genes related to anti-inflammation (14 genes), anti-apoptosis (15 genes), anti-angiogenesis (11 genes) and anti-coagulation (7 genes) [73].

Identification of Active Compounds and Their Metabolites

About 54 main chemical constituents in BYHWD have been identified pharmacologically. They belong to C-glycosyl quinochalcones, flavonoid O-glycosides, isoflavones, monoterpene glycosides, saponins, organic acids and amino acids [93]. To understand the active compounds contributing to the bioactivities of BYHWD, rapid resolution liquid chromatography was coupled with quadrupole time-of-flight tandem mass spectrometry to identify the absorbed components and metabolites in rat urine after oral administration of BYHWD. A total of 50 compounds were detected in rat urine samples within $20 \mathrm{~min}$, including 12 parent compounds and 38 metabolites. Except for 3 prototype components (hydroxysafflor yellow A, paeoniflorin and amygdalin), the identified metabolites mainly came from radix astragali, radix angelicae sinensis, and rhizoma chuanxiong. The results indicated that glucuronidation and sulfation were the major metabolic pathways of isoflavonoids, while glutathione conjugation, glucuronidation and sulfation were the main metabolic pathways of phthalides [69].

A recent study further identified the active compounds of BYHWD by comparing the high-performance liquid chromatography of a drug-containing urine sample with that of a drug-free sample. A total of 17 characteristic compounds were isolated from the methanol extract of a drug-containing urine sample by column chromatography. Their structures, including 11 isoflavanoids, 2 pterocarpanoids and 4 isoflavonoids, were identified by spectroscopic means. Of the 17 compounds, 8 were new compounds. Based on the possible relationship and metabolic pathways of the 17 compounds in vivo, 3R-7, 2'-dihydroxy-3', $4^{\prime}$-dimet hoxyisoflavan(isomucronulatol), 6aR,11aR-3-hydroxy-9,10-dimethoxypterocarpan (methylnissolin, astrapterocarpan), 7,3'-dihydroxy-4'-methoxyisoflavone (calycosin) and 7-hydroxy$4^{\prime}$-methoxyisoflavone (formononetin) were the most important absorptive original isoflavonoid constituents of BYHWD in vivo, which underwent reactions of glucuronidation, hydroxylation, demethylation and reduction [70].

\section{Other Representative TCM Formulas}

MLC901 is a simplified formula from the China State FDA-registered botanical drug MLC601, originally developed from a TCM formula named danqi piantang jiaonang [94], containing radix astragali, radix salvia miltiorrhizae, radix paeoniae rubra, rhizoma chuanxiong, radix angelicae sinensis, $C$. tinctorius, $P$. persica, radix polygalae, and Rhizoma acori tatari- 
nowii. Previously randomized double-blind, controlled trials showed that danqi piantang jiaonang could improve the recovery of neurological functions in stroke patients. MLC901 was reported to increase the proliferation of human embryonic stem cell-derived progenitors [95]. The molecular targets and its active compounds contributing to neurogenesis-promoting activities remain to be addressed. Fuzhi san is a herbal formula used for improving learning and memory. This TCM formula includes Panax ginseng, Scutellaria baicalensis, Acorus talarinowi, and Glycyrrhiza uralensis. A recent study revealed that fuzhi san increased the proliferation of neural progenitor cells and the survival of newborn cells in the hippocampal dentate gyrus of SAMP-8 aging mice and improved learning and memory activities [96]. Liuwei dihuang tang is a classic TCM formula with the herbs of rehmanniae radix, dioscorae radix, corni fructus, alimatis rhizoma, moutan cortex radicis, hoelen, maximowicziae fructus and cervi cornu. The aqueous extract of liuwei dihuang tang revealed to induce the proliferation of rat neural stem cells and increased spatial learning ability by the radial-arm maze test [97]. In addition, kami-ondam-tang (jiawei-wen-dan-tang) is also a potential herbal formula for promoting neurogenesis. The formula consists of the following herbal items: pinelliae rhizoma, bambusae caulis, aurantii immaturus fructus, poria, citri reticulatae pericarpium, glycyrrhizae radix, polygalae radix, scrophulariae radix, ginseng radix, rehmanniae radix, zizyphi spinosae semen, jujubae fructus and zingiberis rhizoma. Kami-ondam-tang treatment increased the doublecortin-positive cells in the hippocampus area and increased step-through latency in the passive avoidance task in mice. The molecular mechanisms are associated with promoting the expressions of pAkt, BDNF and pCREB [98].

\section{Representative Active Compounds from Chinese Herbal Medicine for Promoting Neurogenesis}

EGb761 is a standard extract of Ginkgo biloba, a medicinal herb widely used for the treatments of stroke. Bilobalide is a unique ingredient of Ginkgo biloba, whereas quercetin is its representative antioxidant component. EGb761 and its active compounds bilobalide and quercetin have the ability to promote proliferation of progenitor cells in the rat hippocampus and embryonic brains through activating the CREB signaling pathway $[99,100]$. Salvia miltiorrhiza is a commonly used herb for the treatment of stroke in TCM clinical practices. Salvianolic acid B is one of the active compounds from S. miltiorrhiza. Salvianolic acid B promoted the self-renewal of neural progenitor cells and induced the proliferation of rat embryonic neural stem cells through modulating the PI3K/Akt signaling pathway [101]. In an ischemic stroke rat model, even delayed treatment with salvianolic acid B showed to improve cognitive impairment in post-ischemic rat brain [102]. Baicalin is a flavonoid compound isolated from $S$. baicalensis $G$. Baicalin was previously reported to promote the differentiation of human umbilical cord blood mesenchymal stem cells and rat bone marrow stromal cells into neurons $[103,104]$. Recently, we found that baicalin downregulated p-stat3 and Hes1, and upregulated NeuroD1 and Mash1 (Asc1) in cultured embryonic NSCs. Furthermore, baicalin promoted the neural differentiation but inhibited glial formation, indicating that it could regulate cell fate decision in embryonic NSCs [105]. Tenuigenin and the polygala tenuifolia root extract are isolated and extracted from Polygala tenuifolia, a medicinal herb used for improving insomnia and memory. Recent studies showed that they improved NSC proliferation and neuronal differentiation [106, 107]. Other active compounds from herbal medicine can also be found in the literature, including ginsenosides Rg5 [108], and panax notoginseng saponins [109], wolfberry polysaccharides [110] or epimedium flavonoids [111]. 


\section{Conclusion and Perspectives}

With the development of stem cell biology, targeting adult neurogenesis becomes an important therapeutic strategy for brain repair in post-stroke treatment. Drug discovery from these natural extractions have been paid more attention nowadays with the globally increased popularity of and expenditures on herbal therapies recently. TCM herbs offer a great and unique source of both single compounds and complex combined compounds for drug screening. US and Chinese scientists have jointly established a library of 202 authenticated medicinal plant and fungal species and about 10,000 standard fractions from these materials. More and more compounds and extracts from herbal medicine have been reported to have the potential to induce neurogenesis in the literature. The current progress already opens a door to explore the active compounds from commonly used TCM herbs for stroke treatment and seek their molecular targets. Those discoveries are useful not only for elucidating the scientific basis of herbal medicine for stroke treatment but providing a cue for drug development. However, we must remark that many studies in the literature only reported the phenomenon instead of mechanistic studies. Studies rarely used pharmacological intervention or genetic tools to build the internal relationship between the molecular targets and neurogenesis-promoting effects. How those compounds regulate the signaling and molecule targets, and whether they directly bind those proteins or indirectly affect their activities and expressions remains to be addressed. Overall, Chinese herbal medicine is an important resource for drug discovery and valuable therapeutic strategy to promote adult neurogenesis. Although the precise mechanisms and molecular targets are still unclear, current studies indeed bring novel insights into drug discovery and advancements in fighting post-stroke disability.

Last but not least, we should remark that adult neurogenesis is involved in multiple signal networks; however, much of those are still unknown. The complex networks for controlling neurogenesis make drug development extremely difficult. It is hard to adjust overall abnormal network systems by simply targeting several signaling pathways and molecules. Thus, there is currently no FDA-approved drug for the neuroprotection and neurogenesis. TCM formulas may provide an alternative strategy for this purpose since they generally target multiple signaling pathways. Understanding the synergic effects of different compounds and fractions of herbal formula will be the future direction. With the development of proteomics, metabonomics and bioinformatics, we are at the gate to open the complex and magic world. We believe that the combination of stem cell biology and modern Chinese medicine will lead to the development of novel therapeutic strategies for post-stroke treatment.

\section{Acknowledgements}

The work was supported by grants from the General Research Fund (GRF No. 777610M and No. 776512M), Research Grant Council, Hong Kong SAR; grant from the National Natural Science Foundation of China (No. 31270902), and Seed Funding Programme for Basic Research, The University of Hong Kong (No. 20111159021).

\section{Disclosure Statement}

The authors declare that they have no conflicts of interest. 


\section{References}

1 Feigin VL, et al: Global and regional burden of stroke during 1990-2010: findings from the Global Burden of Disease Study 2010. Lancet 2014;383:245-254.

-2 Mendis S: Stroke disability and rehabilitation of stroke: World Health Organization perspective. Int J Stroke 2013;8:3-4.

3 Liu M, et al: Stroke in China: epidemiology, prevention, and management strategies. Lancet Neurol 2007;6: 456-464.

4 Beal CC: Gender and stroke symptoms: a review of the current literature. J Neurosci Nurs 2010;42:80-87.

5 Mattson MP, et al: Neurodegenerative disorders and ischemic brain diseases. Apoptosis 2001;6:69-81.

6 Murray V, et al: The molecular basis of thrombolysis and its clinical application in stroke. J Intern Med 2010; 267:191-208.

7 Neuroprotection: the end of an era? Lancet 2006;368:1548.

8 Gross CG: Neurogenesis in the adult brain: death of a dogma. Nat Rev Neurosci 2000;1:67-73.

-9 Temple S: The development of neural stem cells. Nature 2001;414:112-117.

10 Alvarez-Buylla A, Garcia-Verdugo JM: Neurogenesis in adult subventricular zone. J Neurosci 2002;22:629634.

11 Cameron HA, McKay RD: Adult neurogenesis produces a large pool of new granule cells in the dentate gyrus. J Comp Neurol 2001;435:406-417.

12 Picard-Riera N, Nait-Oumesmar B, Baron-Van Evercooren A: Endogenous adult neural stem cells: limits and potential to repair the injured central nervous system. J Neurosci Res 2004;76:223-231.

$\checkmark 13$ Tanaka Y, et al: The role of von Hippel-Lindau protein in the differentiation of neural progenitor cells under normoxic and anoxic conditions. Neurosci Lett 2005;383:28-32.

14 Plane JM, et al: Neonatal hypoxic-ischemic injury increases forebrain subventricular zone neurogenesis in the mouse. Neurobiol Dis 2004;16:585-595.

15 Thored P, et al: Long-term neuroblast migration along blood vessels in an area with transient angiogenesis and increased vascularization after stroke. Stroke 2007;38:3032-3039.

16 Macas J, et al: Increased generation of neuronal progenitors after ischemic injury in the aged adult human forebrain. J Neurosci 2006;26:13114-13119.

17 Giusto E, et al: Neuro-immune interactions of neural stem cell transplants: from animal disease models to human trials. Exp Neurol 2013, Epub ahead of print.

18 Lemmens R, Steinberg GK: Stem cell therapy for acute cerebral injury: what do we know and what will the future bring? Curr Opin Neurol 2013;26:617-625.

19 Liu J, et al: Increased neurogenesis in the dentate gyrus after transient global ischemia in gerbils. J Neurosci 1998;18:7768-7778.

20 Rietze R, Poulin P, Weiss S: Mitotically active cells that generate neurons and astrocytes are present in multiple regions of the adult mouse hippocampus. J Comp Neurol 2000;424:397-408.

-21 Mocchetti I, Wrathall JR: Neurotrophic factors in central nervous system trauma. J Neurotrauma 1995;12: 853-870.

22 Schanzer A, et al: Direct stimulation of adult neural stem cells in vitro and neurogenesis in vivo by vascular endothelial growth factor. Brain Pathol 2004;14:237-248.

$\checkmark 23$ Moyse E, et al: Microenvironmental determinants of adult neural stem cell proliferation and lineage commitment in the healthy and injured central nervous system. Curr Stem Cell Res Ther 2008;3:163-184.

-24 Jin K, et al: Vascular endothelial growth factor (VEGF) stimulates neurogenesis in vitro and in vivo. Proc Natl Acad Sci USA 2002;99:11946-11950.

25 Cao L, et al: VEGF links hippocampal activity with neurogenesis, learning and memory. Nat Genet 2004;36: 827-835.

26 Wang Y, et al: VEGF-overexpressing transgenic mice show enhanced post-ischemic neurogenesis and neuromigration. J Neurosci Res 2007;85:740-747.

27 Kim HM, et al: Ex vivo VEGF delivery by neural stem cells enhances proliferation of glial progenitors, angiogenesis, and tissue sparing after spinal cord injury. PLoS One 2009;4:e4987.

-28 Ciccolini F, Svendsen CN: Fibroblast growth factor 2 (FGF-2) promotes acquisition of epidermal growth factor (EGF) responsiveness in mouse striatal precursor cells: identification of neural precursors responding to both EGF and FGF-2. J Neurosci 1998;18:7869-7880.

-29 Raballo R, et al: Basic fibroblast growth factor (Fgf2) is necessary for cell proliferation and neurogenesis in the developing cerebral cortex. J Neurosci 2000;20:5012-5023.

30 Li Q, et al: Modeling the neurovascular niche: VEGF- and BDNF-mediated cross-talk between neural stem cells and endothelial cells: an in vitro study. J Neurosci Res 2006;84:1656-1668.

-31 Lathia JD, Mattson MP, Cheng A: Notch: from neural development to neurological disorders. J Neurochem 2008;107:1471-1481.

32 Androutsellis-Theotokis A, et al: Notch signalling regulates stem cell numbers in vitro and in vivo. Nature 2006;442:823-826.

33 Wang L, et al: The Notch pathway mediates expansion of a progenitor pool and neuronal differentiation in adult neural progenitor cells after stroke. Neuroscience 2009;158:1356-1363. 
Shen et al.: Targeting Neurogenesis: A Promising Therapeutic Strategy for Post-Stroke

-34 Carlen M, et al: Forebrain ependymal cells are Notch-dependent and generate neuroblasts and astrocytes after stroke. Nat Neurosci 2009;12:259-267.

-35 Shen Q, et al: Endothelial cells stimulate self-renewal and expand neurogenesis of neural stem cells. Science 2004;304:1338-1340.

-36 Lanner F, Sohl M, Farnebo F: Functional arterial and venous fate is determined by graded VEGF signaling and notch status during embryonic stem cell differentiation. Arterioscler Thromb Vasc Biol 2007;27:487-493.

-37 Feigenson K, et al: Wnt signaling is sufficient to perturb oligodendrocyte maturation. Mol Cell Neurosci 2009; 42:255-265.

-38 Shimizu T, et al: Wnt signaling controls the timing of oligodendrocyte development in the spinal cord. Dev Biol 2005;282:397-410.

-39 Shruster A, et al: Wnt signaling enhances neurogenesis and improves neurological function after focal ischemic injury. PLoS One 2012;7:e40843.

40 Ruiz i Altaba A, Palma V, Dahmane N: Hedgehog-Gli signalling and the growth of the brain. Nature reviews. Neuroscience 2002;3:24-33.

41 Ingham PW, McMahon AP: Hedgehog signaling in animal development: paradigms and principles. Genes Dev 2001;15:3059-3087.

42 Lai K, et al: Sonic hedgehog regulates adult neural progenitor proliferation in vitro and in vivo. Nat Neurosci 2003;6:21-27.

-43 Machold R, et al: Sonic hedgehog is required for progenitor cell maintenance in telencephalic stem cell niches. Neuron 2003;39:937-950.

44 Balordi F, Fishell G: Hedgehog signaling in the subventricular zone is required for both the maintenance of stem cells and the migration of newborn neurons. J Neurosci 2007;27:5936-5947.

45 Ikezu T, et al: Affinity-purification and characterization of caveolins from the brain: differential expression of caveolin-1, -2, and -3 in brain endothelial and astroglial cell types. Brain Res 1998;804:177-192.

-46 Mikol DD, et al: Schwann cell caveolin-1 expression increases during myelination and decreases after axotomy. Glia 2002;38:191-199.

-47 Arvanitis DN, et al: Membrane-associated estrogen receptor and caveolin-1 are present in central nervous system myelin and oligodendrocyte plasma membranes. J Neurosci Res 2004;75:603-613.

-48 Shen JG, Lee WS, Chen JP, Yang DP: Caveolin-3 peptide protects cardiomyocytes from apoptotic cell death via preserving superoxide dismutase activity and inhibiting caspase-3 activation under hypoxia-reoxygenation. Am J Biomed Sci 2011;3:18.

49 Rothberg KG, et al: Caveolin, a protein component of caveolae membrane coats. Cell 1992;68:673-682.

50 Schwencke $\mathrm{C}$, et al: Caveolae and caveolin in transmembrane signaling: implications for human disease. Cardiovasc Res 2006;70:42-49.

51 Fujita Y, et al: Caveolin-1 in mesangial cells suppresses MAP kinase activation and cell proliferation induced by bFGF and PDGF. Kidney Int 2004;66:1794-1804.

52 Fang K, et al: Overexpression of caveolin-1 inhibits endothelial cell proliferation by arresting the cell cycle at G0/G1 phase. Cell Cycle 2007;6:199-204.

53 Gaudreault SB, et al: A role for caveolin-1 in post-injury reactive neuronal plasticity. J Neurochem 2005;92: 831-839.

54 Kang MJ, Seo JS, Park WY: Caveolin-1 inhibits neurite growth by blocking Rac1/Cdc42 and p21-activated kinase 1 interactions. Neuroreport 2006;17:823-827.

55 Li Y, et al: Caveolin-1 promote astroglial differentiation of neural stem/progenitor cells through modulating Notch1/NICD and Hes1 expressions. Biochem Biophys Res Commun 2011;407:517-524.

56 Li Y, et al: Caveolin-1 inhibits oligodendroglial differentiation of neural stem/progenitor cells through modulating beta-catenin expression. Neurochem Int 2011;59:114-121.

57 Li Y, et al: Caveolin-1 plays a crucial role in inhibiting neuronal differentiation of neural stem/progenitor cells via VEGF signaling-dependent pathway. PLoS One 2011;6:e22901.

58 Mazumdar J, et al: $\mathrm{O}_{2}$ regulates stem cells through Wnt/beta-catenin signalling. Nat Cell Biol 2010;12:10071013.

59 Iguchi $\mathrm{H}$, et al: cAMP response element-binding protein (CREB) is required for epidermal growth factor (EGF)induced cell proliferation and serum response element activation in neural stem cells isolated from the forebrain subventricular zone of adult mice. Endocr J 2011;58:747-759.

60 Peltier J, O'Neill A, Schaffer DV: PI3K/Akt and CREB regulate adult neural hippocampal progenitor proliferation and differentiation. Dev Neurobiol 2007;67:1348-1361.

61 Shioda N, Han F, Fukunaga K: Role of Akt and ERK signaling in the neurogenesis following brain ischemia. Int Rev Neurobiol 2009;85:375-387.

62 Mu Y, Lee SW, Gage FH: Signaling in adult neurogenesis. Curr Opin Neurobiol 2010;20:416-423.

63 Zhang CY, et al: Effects of tianma gouteng fang on transmitter amino acids in the hippocampus extracellular liquids in freely moving rats subjected to brain ischemia. Zhongguo Zhong Yao Za Zhi 2004;29:1061-1065.

-64 Ho SC, et al: Effect of Tianma Gouteng decoction with subtractive ingredients and its active constituents on memory acquisition. Am J Chin Med 2008;36:593-602.

65 Zhao YH, et al: Effect of Yinian Jiangya Yin on primary hypertension in early stage - a clinical observation on 40 patients. J Tradit Chin Med 2010;30:171-175. 
Zhang HW, et al: Tianma Gouteng Yin formula for treating primary hypertension. Cochrane Database Syst Rev 2012;6:CD008166.

67 Wei RL, et al: A systematic review and meta-analysis of buyang huanwu decoction in animal model of focal cerebral ischemia. Evid Based Complement Alternat Med 2013;2013:138484.

68 Hao CZ, et al: Clinical efficacy and safety of buyang huanwu decoction for acute ischemic stroke: a systematic review and meta-analysis of 19 randomized controlled trials. Evid Based Complement Alternat Med 2012; 2012:630124.

69 Wen XD, et al: Identification of metabolites of Buyang Huanwu decoction in rat urine using liquid chromatography-quadrupole time-of-flight mass spectrometry. J Pharm Biomed Anal 2012;67-68:114-122.

-70 Yang DH, et al: Absorptive constituents and their metabolites in drug-containing urine samples from Wuzhishan miniature pigs orally administered with Buyang Huanwu decoction. J Nat Med 2014;68:11-21.

-71 Tong L, Tan XH, Shen JG: Comparative study of Buyang Huanwu decoction and the different combinations of its ingredients on neurogenesis following ischemic stroke in rats. Zhongguo Zhong Xi Yi Jie He Za Zhi 2007;27: 519-522.

-72 Cai G, et al: Buyang Huanwu decoction can improve recovery of neurological function, reduce infarction volume, stimulate neural proliferation and modulate VEGF and Flk1 expressions in transient focal cerebral ischaemic rat brains. J Ethnopharmacol 2007;113:292-299.

73 Wang HW, et al: Deciphering the neuroprotective mechanisms of Bu-yang Huan-wu decoction by an integrative neurofunctional and genomic approach in ischemic stroke mice. J Ethnopharmacol 2011;138:22-33.

74 Wu Y, Jiang L: Clinical study on buyang huanwu decoction to the metabolic imbalance of endothelin and calcitonin gene related peptide in patients with early cerebral infarction. Zhongguo Zhong Xi Yi Jie He Za Zhi 1998; 18:396-398.

75 Zhao LD, et al: Neuroprotective effect of Buyang Huanwu decoction against focal cerebral ischemia/reperfusion injury in rats - time window and mechanism. J Ethnopharmacol 2012;140:339-344.

76 Liao CL, Tong L, Chen YY: Effect of Buyanghuanwu decoction on neuronal nitric oxide synthase expression after permanent focal cerebral ischemia in rats. Di Yi Jun Yi Da Xue Xue Bao 2004;24:864-886, 891.

$\checkmark 7$ Qu HD, et al: Protective effect of Buyanghuanwu Tang on cultured rat cortical neurons against hypoxiainduced apoptosis. Di Yi Jun Yi Da Xue Xue Bao 2002;22:35-38.

78 Qu HD, Tong L, Shen JG: Effect of buyang huanwu decoction drug serum on expression of p53 and p21 genes in cultured rat's cerebral cortical neuron after hypoxia in vitro. Zhongguo Zhong Xi Yi Jie He Za Zhi 2004;24: 133-135.

79 Li XM, et al: Neuroprotective effects of Buyang Huanwu decoction on neuronal injury in hippocampus after transient forebrain ischemia in rats. Neurosci Lett 2003;346:29-32.

-80 Deng CQ, Wang M, He FY: Effect of buyang huanwu decoction and its active regions combination on brain heat shock protein 70 expression in gerbils after cerebral ischemia/reperfusion. Zhongguo Zhong Xi Yi Jie He Za Zhi 2002;22:193-195, 210.

-81 Yi J, et al: Effect of Buyang Huanwu decoction on interleukin-1beta and tumor necrosis factor-alpha expression in rats after cerebral infarction. Zhongguo Wei Zhong Bing Ji Jiu Yi Xue 2010;22:599-601.

-82 Tang YH, Li H, Chen BY: Effect of active fraction of buyang huanwu decoction on caspase expression in rats after focal cerebral ischemic reperfusion. Zhongguo Zhong Xi Yi Jie He Za Zhi 2006;26:533-537.

-83 Sun J, et al: Buyang Huanwu decoction promotes growth and differentiation of neural progenitor cells: using a serum pharmacological method. J Ethnopharmacol 2007;113:199-203.

84 Wang, L, et al: Effect of Buyang Huanwu decoction on amino acid content in cerebrospinal fluid of rats during ischemic/reperfusion injury. J Pharm Biomed Anal 2013;86:143-150.

85 Li AG, He SL, Pen YG: Effect of buyang huanwu decoction on the antithrombotic functions of the vessel wall. Zhong Xi Yi Jie He Za Zhi 1989;9:545-547, 517.

-86 Zhao, Y.N, et al: Effect of BuYangHuanWu recipe on cerebral microcirculation in gerbils with ischemia-reperfusion. Sichuan Da Xue Xue Bao Yi Xue Ban 2010;41:53-56.

87 Zhang J, Li C, Zhang Y: Experimental study on effect of Buyang Huanwu decoction on arterial blood platelet activating factor content pre- and post-arterial thrombosis of rats. Zhongguo Zhong Xi Yi Jie He Za Zhi 1998; 18:730-732.

88 Tong L, et al: Effects of Buyanghuanwu decoction (BYHWT) on proliferation of cultured rat cortical neurons. Di Yi Jun Yi Da Xue Xue Bao 2002;22:711-712.

89 Cai GX, Liu BY: Effect of ultra-micronized Buyang Huanwu decoction on neurological function, quality of life, and serum vascular endothelial growth factor in patients convalescent from cerebral infarction. Zhongguo Wei Zhong Bing Ji Jiu Yi Xue 2010;22:591-594.

90 Kong X, et al: The neuroprotective effect of Buyang Huanwu decoction on rat ischemic/reperfusion brain damage by promoting migration of neural precursor cells. Rejuvenation Res 2013, Epub ahead of print.

91 Zhang YK, Han XY, Che ZY: Effects of buyang huanwu tang combined with bone marrow mesenchymal stem cell transplantation on the expression of VEGF and Ki-67 in the brain tissue of the cerebral ischemia-reperfusion model rat. J Tradit Chin Med 2010;30:278-282.

92 Li TJ, et al: DNA microarray analysis of protective mechanism of buyang huanwu decoction on focal cerebral ischemia/reperfusion in rats. Zhongguo Zhong Yao Za Zhi 2004;29:559-563.

$\$ 93$ Liu EH, et al: Rapid separation and identification of 54 major constituents in Buyang Huanwu decoction by ultra-fast HPLC system coupled with DAD-TOF/MS. Biomed Chromatogr 2009;23:828-842. 
Shen et al.: Targeting Neurogenesis: A Promising Therapeutic Strategy for Post-Stroke Treatment with Chinese Herbal Medicine

94 Chen C, et al: Danqi Piantang Jiaonang (DJ), a traditional Chinese medicine, in poststroke recovery. Stroke 2009;40:859-863.

95 Heurteaux C, et al: Neuroprotective and neuroproliferative activities of NeuroAid (MLC601, MLC901), a Chinese medicine, in vitro and in vivo. Neuropharmacology 2010;58:987-1001.

-96 Yang H, et al: Effects of Chinese herbal medicine Fuzhisan on autologous neural stem cells in the brain of SAMP-8 mice. Exp Gerontol 2011;46:628-636.

97 Cragg GM, et al: Ethnobotany and drug discovery: the experience of the US National Cancer Institute. Ciba Found Symp 1994;185:178-196.

98 Hong JG, et al: The memory-enhancing effects of Kami-ondam-tang in mice. J Ethnopharmacol 2011;137:251256.

99 Tchantchou F, et al: Stimulation of neurogenesis and synaptogenesis by bilobalide and quercetin via common final pathway in hippocampal neurons. J Alzheimers Dis 2009;18:787-798.

100 Tchantchou F, et al: EGb 761 enhances adult hippocampal neurogenesis and phosphorylation of CREB in transgenic mouse model of Alzheimer's disease. FASEB J 2007;21:2400-2408.

101 Zhuang P, et al: Direct stimulation of adult neural stem/progenitor cells in vitro and neurogenesis in vivo by salvianolic acid B. PLoS One 2012;7:e35636.

102 Guo G, et al: Effects of salvianolic acid B on proliferation, neurite outgrowth and differentiation of neural stem cells derived from the cerebral cortex of embryonic mice. Sci China Life Sci 2010;53:653-662.

103 Jia Y, et al: Differentiation of rat bone marrow stromal cells into neuron induced by baicalin. Zhonghua Yi Xue Za Zhi 2002;82:1337-1341.

104 Yan XH, Huang RB: Differentiation of human umbilical cord blood mesenchymal stem cells toward neurons induced by baicalin in vitro. Zhonghua Er Ke Za Zhi 2006;44:214-219.

105 Li Y, et al: Baicalin promotes neuronal differentiation of neural stem/progenitor cells through modulating p-stat3 and bHLH family protein expression. Brain Res 2012;1429:36-42.

106 Chen Y, et al: Tenuigenin promotes proliferation and differentiation of hippocampal neural stem cells. Neurochem Res 2012;37:771-777.

107 Park HJ, et al: Effects of Polygala tenuifolia root extract on proliferation of neural stem cells in the hippocampal CA1 region. Phytother Res 2008;22:1324-1329.

108 Liu JW, et al: Panaxadiol glycosides that induce neuronal differentiation in neurosphere stem cells. J Nat Prod 2007;70:1329-1334.

109 Si YC, et al: Effects of Panax notoginseng saponins on proliferation and differentiation of rat hippocampal neural stem cells. Am J Chin Med 2011;39:999-1013.

110 Lau BW, et al: Polysaccharides from wolfberry prevents corticosterone-induced inhibition of sexual behavior and increases neurogenesis. PLoS One 2012;7:e33374.

111 Yao R, et al: Effects of Epimedium flavonoids on proliferation and differentiation of neural stem cells in vitro. Neurol Res 2010;32:736-742. 